A l'intersection des discours de spécialité :

hétérogénéité et unité

\title{
Grammaire et degré de spécialisation
}

\section{Elsa Pic et Grégory Furmaniak}

\section{(2) OpenEdition}

Journals

Édition électronique

URL : http://journals.openedition.org/asp/1811

DOI : $10.4000 /$ asp. 1811

ISBN : 978-2-8218-0414-2

ISSN : 2108-6354

\section{Éditeur}

Groupe d'étude et de recherche en anglais de spécialité

\section{Édition imprimée}

Date de publication : 30 novembre 2010

Pagination : $39-55$

ISSN : 1246-8185

Référence électronique

Elsa Pic et Grégory Furmaniak, « Grammaire et degré de spécialisation », ASp [En ligne], 58 | 2010, mis en ligne le 30 novembre 2013, consulté le 03 novembre 2020. URL : http://journals.openedition.org/ asp/1811 ; DOI : https://doi.org/10.4000/asp.1811

Ce document a été généré automatiquement le 3 novembre 2020.

Tous droits réservés 


\title{
Grammaire et degré de spécialisation
}

\author{
Elsa Pic et Grégory Furmaniak
}

\section{Introduction}

1 Depuis Barber (1962), de nombreux chercheurs se sont penchés sur les spécificités grammaticales des langues de spécialité (cf. Halliday, McIntosh \& Strevens 1964, Huddleston 1971, Banks 1993, Magnet 2001, Richard 2003, etc.).

2 Certains auteurs (Adams Smith 1984, Swales 1990, Bhatia 1993) estiment cependant que les langues de spécialité recouvrent des réalités trop hétéroclites pour être caractérisées en termes exclusivement linguistiques. Swales, par exemple, juge que :

Even if there remains some shorthand convenience attached to retaining registral labels such as scientific, medical, or even newspaper English, in reality such terms can now be seen to be systematically misleading. They overprivilege a homogeneity of content at the expense of variation in communicative purpose, addresser-addressee relationship and genre conventions. (Swales $1990: 3$ )

Dans cette optique, il ne s'agit plus de classer les textes en fonction de leur thématique mais du type d'événement communicationnel auxquels ils participent. Les genres ainsi dégagés (abstract, article scientifique, recension d'article, etc.) peuvent être considérés comme autant de discours transversaux. Ils ne constituent pas des sous-types de langues de spécialité, ils les transcendent.

4 La transversalité dont il sera question ici n'est pas strictement générique ${ }^{1}$. À la suite de Rondeau (1983) et de Resche (2001), nous reconnaissons qu'une langue de spécialité peut être plus ou moins spécialisée. Il est alors théoriquement légitime de postuler des langues transversales indépendantes de la discipline et définies seulement par leur degré de spécialisation (par exemple, la langue vulgarisée, qu'elle soit employée par le mathématicien, l'historien ou le juriste).

5 L'un des objectifs de cet article est de vérifier si les langues transversales ainsi définies ont un fondement empirique. En particulier, nous nous demanderons si, au-delà des 
disciplines (et donc des langues de spécialité), il est possible de dégager des propriétés grammaticales propres au degré de spécialisation indépendamment du contenu disciplinaire.

6 En plus d'aborder les langues de spécialité sous un angle transversal relativement neuf, notre approche se distingue de la recherche contemporaine sur plusieurs points. Tout d'abord, nous nous intéressons aux langues des sciences humaines (la philosophie et l'histoire), domaine parfois négligé par la recherche hexagonale qui s'est focalisée sur les sciences dures et le droit. En outre, contrairement à la perspective majoritairement quantitative des travaux sur la grammaire des langues de spécialité, nous associons aux mesures quantitatives des analyses qualitatives aussi fines que possible. Il nous semble en effet que, pour intéressantes qu'elles soient, les spécificités quantitatives sont trop générales pour être exploitées dans le cadre d'une tentative de caractérisation d'un type de discours particulier.

\section{Définitions et hypothèse}

7 Nous n'entrons pas pour les besoins de cet article dans la discussion portant sur les dénominations " langue de spécialité », « discours de spécialité », « langage spécialisé » ou «LSP » et emploierons indifféremment ces termes pour référer au discours sur la discipline par la communauté des experts. Néanmoins, nous nous fondons sur un certain nombre de consensus à leur propos qu'il est nécessaire de rappeler. Sur le plan grammatical, les langues de spécialité ne constituent pas un système linguistique à part, mais une utilisation particulière de ce système. Comme le formulent Sager, Dungworth et McDonald (1980:38) :

The nature of language is such that general language and special languages can be accommodated within one natural language: the fundamental characteristics of language are manifested both in English and in the language of chemical engineering, both in French and in the language of physics. The differences between general and special languages is a difference of degree rather than kind: the degree to which the fundamental characteristics of language are maximised or minimized in special languages.

8 Ainsi certains marqueurs sont plus fréquents dans une langue de spécialité que dans la langue générale (à l'instar du passif, récurrent en anglais juridique), ou à l'inverse absents d'un discours spécialisé (on trouve très peu de prétérits en be -ing dans l'anglais de la philosophie). Les valeurs de ces marqueurs peuvent aussi se singulariser par rapport aux valeurs qu'ils ont en langue générale ${ }^{2}$ (par exemple, si on trouve bien des modaux en anglais juridique, les valeurs épistémiques sont souvent rares ou absentes.)

9 Partant du postulat que les discours de spécialité ne sont pas homogènes et qu'ils comprennent différents degrés de spécialisation, nous avancerons l'hypothèse qu'il existe des variations grammaticales non seulement entre les discours de spécialités différentes, mais aussi entre les divers degrés de spécialisation au sein d'une même discipline. Nous chercherons donc à vérifier s'il existe des grammaires transversales liées au degré de spécialisation indépendamment de la discipline. En d'autres termes, peut-on identifier une grammaire de la vulgarisation différente d'une grammaire de l'expert?

10 Si notre hypothèse est avérée, il faut s'attendre à ce que ces grammaires transversales se distinguent entre elles au niveau des marqueurs intersubjectifs, qui codent les liens 
entre auteur et lecteur, comme les pronoms personnels, les modaux, le passif, les clivées, etc. Les discours disciplinaires, eux, se démarqueraient sur le plan grammatical au travers de marqueurs plus référentiels - ou plutôt, à dominante référentielle, comme le prétérit en be-ing ou le pluperfect.

11 Cette distinction entre marqueurs à dominante référentielle ou intersubjective s'appuie sur les théories fonctionnalistes (cf. Jakobson 1963, Hyme 1966, Hagège 1990, Halliday 1994) qui considèrent que les énoncés ne peuvent pas être analysés sans une prise en compte des fonctions (ou méta-fonctions ${ }^{3}$ ) qu'ils remplissent. Bien que le nombre et la nature de ces méta-fonctions varient d'un auteur à l'autre, tous s'accordent pour dégager une méta-fonction que l'on qualifiera, à la suite de Jakobson (1963:220) de référentielle. Elle est "sémantico-référentielle» chez Hagège (1990: 29) et « expérientielle » chez Halliday (1994: 36) mais il s'agit toujours de considérer l'énoncé pour ce qu'il dit du monde, pour sa capacité à construire une représentation de l'extralinguistique («Language enables human beings to build a mental picture of reality » Halliday 1994: 106) et à la communiquer. La fonction intersubjective telle que nous la définissons inclut, d'une part, les fonctions "émotive " et "conative " de Jakobson (ibid.), regroupées, chez Halliday (ibid.), au sein de la méta-fonction " interpersonnelle». La fonction émotive (ou expressive) est "centrée sur le destinateur [et] vise à une expression directe de l'attitude du sujet à l'égard de ce dont il parle" (Jakobson 1963: 214), tandis que la fonction conative est définie comme " $[1$ '] orientation vers le destinataire » (ibid. 216). D'autre part, relève également, selon nous, de la méta-fonction intersubjective l'organisation thème/rhème des énoncés, c'est-à-dire le "point de vue énonciatif-hiérarchique » de Hagège (1990:52) et la fonction « textuelle » de Halliday (1994 : 36). Ce regroupement est motivé par le fait que la structure informationnelle d'un énoncé est intimement liée au point de vue du destinateur et à ce qu'il pense être l'état des connaissances du destinataire.

12 En proposant une distinction entre marqueurs référentiels et intersubjectifs, nous postulons que, même si les méta-fonctions que nous avons retenues sont réalisées au niveau de l'énoncé, un marqueur ou une construction peut contribuer individuellement à une ou à plusieurs méta-fonctions. C'est ce que souligne Halliday (ibid. 35) :

[...] not everything has function in every dimension of structure; for example, some

parts of the clause (e.g. perhaps) play no role in the clause as representation.

Cela implique qu'une forme linguistique donnée ne se limite pas nécessairement à un seul niveau fonctionnel (cf. par exemple Delmas 1993 sur la dimension à la fois aspectuelle, donc plutôt référentielle, et intersubjective de be-ing). C'est pour cette raison que la typologie des constructions grammaticales que nous proposons se formule en termes de dominante référentielle ou intersubjective.

\section{Méthode}

\subsection{Corpus}

14 Notre hypothèse de grammaires sensibles au degré de spécialisation a été confrontée à la réalité des textes par le biais d'une étude de corpus. Nous avons constitué un corpus de 100000 mots, divisé en deux sous-corpus disciplinaires: l'un historique, l'autre philosophique. Chacun de ces deux sous-corpus est lui-même stratifié en corpus spécialisé et corpus de vulgarisation de la discipline. 
britanniques à comité de lecture. Il s'agit de The Historian et de The Historical Journal pour le corpus historique, et de Minerva - An Internet Journal of Philosophy, de Analysis et de Metaphilosophy pour le corpus philosophique. Écrits par et pour des chercheurs, ces publications universitaires reconnues se donnent pour objectif d'être à la pointe de la recherche dans leur domaine :

As The Historian celebrates its $71^{\text {st }}$ year of continuous publication, the journal continues to publish a diverse collection of high-quality original articles and debate on historical scholarship and current historiography. [...] With more than 13,000 individual and institutional subscribers, The Historian brings the many strands of historical analysis together in one authoritative journal. (<http://www.wiley.com/ bw/journal.asp?ref=0018-2370>)

21 Les textes non spécialisés ont été sélectionnés dans des magazines (également britanniques) de vulgarisation à destination du grand public. Nous nous sommes efforcés de choisir des sources comparables quant à leur degré de vulgarisation, en nous fondant en particulier sur la ligne éditoriale et le public cible des revues. History Today, notre source vulgarisée en histoire, affiche comme ambition de toucher le plus grand nombre, d'informer et de divertir de façon accessible :

History Today is a unique cultural institution, bringing the best in historical writing and research to a wide audience. 
[...] The magazine created the concept of popular history, mixing styles, genres and periods to achieve a fusion of intellectual excitement and readability. All are carefully edited and illustrated to make the magazine a pleasurable, as well as an informative, read. (<http://www.historytoday.com/NewBlank.aspx?m=6325>) temporels, puisqu'ils ont pour rôle principal de situer un procès dans le temps et, plus précisément, par rapport à un point de référence temporel. La quasi-absence du temps (tense) présent dans les textes historiques et la très faible fréquence du prétérit en -ing dans le corpus philosophique nous ont conduits à retenir, comme marqueur référentiel, le pluperfect. Pour ce qui est des marqueurs intersubjectifs, notre choix s'est porté sur trois modaux (le lien entre modalité et intersubjectivité n'est plus à démontrer), en l'occurrence, may et son passé morphologique might, ainsi que must. Ces auxiliaires sont en effet unanimement considérés comme nettement plus intersubjectifs que can/could et will/would (cf. Adamczewski 1982). Quant à shall/should, ils sont trop rares dans notre corpus pour fournir des résultats exploitables.

Think, notre source vulgarisée en philosophie, sous-titrée Philosophy for everyone, vise les mêmes objectifs :

Think demonstrates the relevance of philosophy to everyday life and forges a direct link between contemporary philosophy and the widest possible readership. The journal is written in an engaging and straightforward way, but raises fundamental questions about philosophical issues of topical importance. [...] Think is aimed at a very wide audience encompassing undergraduates, schools, colleges and the general public. (http://journals.cambridge.org/action/displayJournal?jid=THI) sont pas les mêmes thèmes qui sont abordés dans les deux types de sources. En philosophie en particulier, il s'est avéré impossible de trouver les mêmes notions traitées dans les revues universitaires et dans le magazine de vulgarisation. On peut considérer cette asymétrie comme problématique mais on peut aussi estimer qu'elle reflète une réalité (les textes vulgarisés ne traitent généralement pas les mêmes sujets que les textes spécialisés) qui serait trahie par la recherche d'un équilibre thématique artificiel.

d'élargir les possibilités d'exploitation du concordancier utilisé, Antconc 3.2.15.

Si l'emploi du concordancier sur texte étiqueté nous permet de procéder à des analyses quantitatives rapides et de grande ampleur, que ce soit via la recherche par mots clés (ex. : modaux) ou par tags (ex: passifs, pluperfect), les analyses qualitatives doivent rester « manuelles ». Chacune des occurrences des marqueurs retenus pour analyse est examinée individuellement pour décider de son classement dans telle ou telle valeur.

plus haut, et les typologies permettant leur classement, sont maintenant présentés.

\subsection{Typologie des marqueurs}

La typologie sémantique des marqueurs, nécessaire à l'analyse qualitative du corpus, est largement inspirée de Larreya \& Rivière (2005), mises à part quelques adaptations mineures. 

réference passé (noté $\mathrm{T}_{-1}$ ) et pertinent en $\mathrm{T}_{-1}$

(1) He had never had occasion to confront it as what William James called a 'live option'.

31 Dans sa valeur de continuité (cf. exemple 2), le pluperfect renvoie à un procès passé (état ou habitude) commencé avant $\mathrm{T}_{-1}$ et toujours vrai en $\mathrm{T}_{-1}$.

(2) These Memoirs had been in the public domain for seven years when the publisher Henry Colburn announced that a new anonymous memoir was being prepared for print.

La valeur d'antériorité est illustrée en (3). Il s'agit toujours de situer un procès comme antérieur au moment $\mathrm{T}_{-1}$ mais, contrairement à l'emploi résultatif, aucun lien autre que temporel n'est impliqué entre ce procès et le moment de référence.

(3) Marcus could not overcome a hankering for the more varied and interesting life that Dr. Smythson had provided for him while he was in his case.

La valeur d'irréel, qui apparaît en (4), correspond à un procès passé contrefactuel (c'està-dire qui n'a pas eu lieu).

(4) We all tend to think that had we been in charge of creation, frankly we would have done it better.

Enfin, l'utilisation du pluperfect peut être déclenchée par le phénomène de concordance des temps. Ce cas de figure, illustré par (5), s'observe essentiellement dans les subordonnées nominalisées régies par un verbe au prétérit qui vient poser un repère temporel passé et qui oblige le destinateur à recourir au pluperfect pour tout procès antérieur à ce repère.

(5) I see. I had no idea that such Brain-O-Matic machines had been developed. Concernant les modaux, nous conservons l'opposition traditionnelle radical/ épistémique. La modalité radicale concerne les conditions de réalisation du procès (permission, capacité, obligation, volonté, etc.). La modalité épistémique correspond à l'expression d'un degré de croyance vis-à-vis de la réalité du procès.

Nous proposons, pour may, deux valeurs radicales principales. Dans le premier cas, illustré en (6), may exprime une permission qui, dans notre corpus, prend souvent la forme d'une légitimité intellectuelle. L'exemple (6) peut en effet être glosé par «il est permis/légitime d'objecter que...»

(6) It may be objected that 'possible' is not vacuous since its negation is not, since we can say a round square is 'not possible'.

Might possède une valeur assez proche, comme on peut l'observer en (7), même si la forme passée a souvent tendance à brouiller la valeur du modal dans le domaine du radical.

(7) So, if we think about the existence or apparent design of the world, for example, we might say that these are effects which need to be explained by the agency of a powerful and probably benevolent cause.

La valeur sporadique, en (8), permet de renvoyer à un procès qui se réalise de façon plus ou moins régulière. Dans notre corpus, nous n'avons trouvé aucune occurrence de might correspondant à cet emploi. 
(8) According to deontology, actions are right or wrong because they obey some rule or other. That rule may mention consequences, but it may not. à-dire impliqué par - un autre procès ou une situation donnée. En (15), par exemple, le fait que Dieu soit un esprit désincarné est la conséquence logique de l'hypothèse construite par la subordonnée.

(15) If God does not have a body, he must be a disembodied mind who exists in another world but brings about changes in this world.

47

may have acted for personal profit.

(10) Cardinal John O'Connor authorized a study of Sheen's life as the first step in a process that might result in the canonization of this famed Cold War protagonist.

La valeur de concession qu'on trouve en (11-12) est également partagée par may et might. Elle regroupe les emplois du type $\mathrm{x}$ may/might be true, but/yet $\mathrm{y}$ is not, ainsi que les occurrences de may ou might au sein de subordonnées dites concessives introduites par (al)though, whatever, however, no matter, etc.

(11) Although his writing may not achieve the "writerly" complexity of a Cavell, it is central to Rorty's philosophical achievement that he writes in the specific ways that he does.

(12) I might be justified in missing my niece's birthday party in order to get extra work done over the weekend, but I am not justified in killing my boss so as to take his place.

Il s'agit à chaque fois de concéder la véracité de la proposition modalisée tout en l'écartant comme non pertinente.

Pour ce qui est de must, on retiendra les valeurs suivantes.

Le modal exprime une obligation en (13). Une pression s'exerce sur le référent du sujet afin qu'il accomplisse l'action dénotée par le prédicat.

(13) And we must care about the education received by all children, not just those capable of academic excellence.

Dans sa valeur de nécessité, must indique que le procès est souhaitable relativement à un but donné (explicite ou non) sans idée de contrainte sur le référent du sujet. Soit l'exemple (14) :

(14) If God's person-like characteristics must be understood in a metaphorical sense, we cannot draw a clear line between a personal god and an impersonal Absolute.

Les emplois épistémiques de must se divisent en deux sous-catégories.

La valeur implicative correspond à un procès vu comme découlant logiquement - c'est(16), en revanche, l'énonciateur infère, à partir des indices dont il dispose, la forte probabilité que le procès ait eu lieu.

(16) Geddes and Wollstonecraft were the workhorses of the early issues, their (anonymous) contributions being so numerous that they must both practically have been in Johnson's full-time pay. 


\section{Résultats} (215 occurrences) qu'en philosophie (37 occurrences). On note aussi que les emplois de ce marqueur diffèrent entre les deux disciplines : la valeur d'irréel par exemple est quasiment inexistante en histoire alors qu'elle représente un tiers des emplois du pluperfect en philosophie. À l'inverse, les valeurs de continuité et de concordance des temps sont absentes en philosophie, mais non négligeables en histoire. Les valeurs plus fréquentes (résultatif et passé antérieur) sont représentées dans les mêmes ordres de grandeur dans les deux corpus.

Tableau 1. Les valeurs du pluperfect selon la discipline et son degré de spécialisation

\begin{tabular}{|l||c|c|c|c||c|c|c|c|}
\hline \multicolumn{1}{|c||}{} & \multicolumn{4}{c||}{ PHILOSOPHIE } & \multicolumn{4}{c|}{ HISTOIRE } \\
\cline { 2 - 10 } & SPÉCIALISÉ & \multicolumn{1}{l|}{ VULGARISÉ } & & SPÉCIALISÉ & & VULGARISÉ & \\
\hline $\begin{array}{l}\text { Total } \\
\text { pluperfect }\end{array}$ & 9 & $\%$ & 28 & $\%$ & 107 & $\%$ & 108 & $\%$ \\
\hline Résultatif & 1 & 11,1 & 14 & 50,0 & 30 & 28 & 41 & 38 \\
\hline Continuité & 0 & 0,0 & 0 & 0,0 & 11 & 10,3 & 19 & 17,7 \\
\hline $\begin{array}{l}\text { Passé } \\
\text { antérieur }\end{array}$ & 5 & 55,6 & 4 & 14,3 & 50 & 46,8 & 39 & 36,1 \\
\hline Irréel & 3 & 33,3 & 9 & 32,1 & 1 & 0,9 & 3 & 2,7 \\
\hline $\begin{array}{l}\text { Concordance } \\
\text { des temps }\end{array}$ & 0 & 0,0 & 1 & 3,6 & 15 & 14 & 6 & 5,5 \\
\hline
\end{tabular}

Il semble donc que le pluperfect soit un marqueur effectivement plus sensible à la discipline qu'au degré de spécialisation. On trouve en effet le même nombre d'occurrences du pluperfect en histoire spécialisée (107) qu'en histoire vulgarisée (108). Les emplois sont comparables en histoire spécialisée et en histoire vulgarisée.

Les résultats concernant les modaux sont présentés dans les tableaux 2 et 3 . On observe que le discours philosophique, quel que soit son degré de spécialisation, est toujours plus "modalisé » que le discours historique. Sur le plan des valeurs, il est difficile d'apprécier si certains emplois sont propres au discours vulgarisé ou au discours spécialisé : pour may, la concession domine légèrement dans les deux discours de vulgarisation; l'épistémique l'emporte dans les deux discours spécialisés. Pour might, aucune tendance commune ne se dégage, les résultats étant même contradictoires d'un discours de spécialité à l'autre, d'un discours de vulgarisation à l'autre.

Tableau 2. Les valeurs de may et might selon la discipline et son degré de spécialisation

\begin{tabular}{|l||c|c|c|c||c|c|c|c|}
\hline \multicolumn{1}{|c||}{} & \multicolumn{3}{c||}{ PHILOSOPHIE } & \multicolumn{4}{c|}{ HISTOIRE } \\
\cline { 2 - 10 } & SPÉCIALISÉ & & \multicolumn{1}{c|}{ VULGARISÉ } & & SPÉCIALISÉ & & VULGARISÉ & \\
\hline Total MAY & 20 & $\%$ & 37 & $\%$ & 11 & $\%$ & 12 & $\%$ \\
\hline Radical & 6 & 30,0 & 15 & 40,5 & 0 & 0 & 3 & 25 \\
\hline Epistémique & 11 & 55,0 & 14 & 37,8 & 11 & 100 & 7 & 58,4 \\
\hline Concession & 3 & 15,0 & 8 & 21,6 & 0 & 0 & 2 & 16,6 \\
\hline & & & & & & & & \\
\hline Total MIGHT & 42 & $\%$ & 46 & $\%$ & 13 & $\%$ & 16 & $\%$ \\
\hline Radical & 18 & 42,9 & 5 & 10,9 & 3 & 23 & 2 & 12,5 \\
\hline Epistémique & 21 & 50,0 & 38 & 82,6 & 10 & 76,9 & 10 & 62,5 \\
\hline Concession & 3 & 7,1 & 3 & 6,5 & 0 & 0 & 4 & 25 \\
\hline
\end{tabular}


52 L'étude des valeurs de must révèle les mêmes tendances systématiquement contradictoires entre le discours spécialisé de la philosophie et le discours spécialisé de l'histoire, et entre les discours de vulgarisation des deux disciplines.

Tableau 3. Les valeurs de must selon la discipline et son degré de spécialisation

\begin{tabular}{|l||c|c|c|c||c|c|c|c|}
\hline \multicolumn{1}{|c||}{} & \multicolumn{4}{c||}{ PHILOSOPHIE } & \multicolumn{4}{c|}{ HISTOIRE } \\
\cline { 2 - 10 } & SPÉCIALISÉ & & VULGARISÉ & & SPÉCIALISÉ & & VULGARISÉ & \\
\hline Total MUST & 31 & $\%$ & 31 & $\%$ & 15 & $\%$ & 6 & $\%$ \\
\hline Obligation & 18 & 58,1 & 17 & 54,8 & 11 & 73,3 & 5 & 83,3 \\
\hline Nécessité & 7 & 22,6 & 4 & 12,9 & 1 & 6,6 & 1 & 16,7 \\
\hline Implication & 6 & 19,4 & 7 & 22,6 & 2 & 13,3 & 0 & 0 \\
\hline Probabilité & 0 & 0 & 5 & 16,1 & 1 & 6,6 & 0 & 0 \\
\hline $\begin{array}{l}\text { Total } \\
\text { épistémique }\end{array}$ & 6 & 19,4 & 12 & 38,7 & 3 & 20 & 0 & 0 \\
\hline
\end{tabular}

\section{Interprétation des résultats}

Ces résultats mettent au jour des différences considérables entre les disciplines. Le fait n'est pas nouveau mais il mérite d'être relevé. La remise en cause, au sein des genre studies (cf. Swales supra) de la notion même de langue de spécialité ne se vérifie pas dans notre corpus. La grammaire est bel et bien sensible au domaine disciplinaire. Plus précisément, c'est, comme on pouvait s'y attendre, au niveau des marqueurs référentiels que les spécificités grammaticales de chaque langue de spécialité sont les plus visibles. La tendance que l'on avait déjà observée avec le prétérit en -ing (7 occurrences seulement en philosophie contre 22 en histoire) est ainsi confirmée avec le pluperfect. L'explication est évidente. Les données montrent que, relativement au nombre total de formes passées, la proportion de pluperfect est sensiblement identique dans les deux disciplines $(7,5 \%$ des formes passées en philosophie contre $9 \%$ en histoire). La rareté du prétérit en -ing et du pluperfect en philosophie et leur fréquence élevée en histoire ne font que refléter une tendance plus générale, à savoir, le recours beaucoup plus systématique aux temps du passé en histoire (2 422 occurrences contre 496 en philosophie). Il n'y a là rien de surprenant. Il s'agit néanmoins d'une composante importante de la grammaire de l'historien et il serait absurde de ne pas la prendre en compte au motif de son caractère évident et attendu.

Pour ce qui est des marqueurs intersubjectifs, rappelons que l'hypothèse envisagée était la suivante. S'il est possible de dégager des propriétés grammaticales propres aux discours transversaux définis sur la base de leur degré de spécialisation, il est plus que probable que celles-ci se manifestent plutôt du côté des marqueurs intersubjectifs, dans la mesure où ces derniers sont censées marquer le rapport auteur/lecteur qui est lié au degré de spécialisation.

Force est de constater que cette hypothèse ne trouve pas, dans notre corpus, une confirmation éclatante. Nous l'avons observé en particulier avec might, et de façon incontestable avec must. Nous pensions par exemple que le discours vulgarisé, qu'il soit philosophique ou historique, ferait la même utilisation de must. De même, nous prévoyions que le discours spécialisé, quelle que soit la discipline, présenterait des similitudes au niveau de ses emplois de might. Il n'en est rien. Pour may, nous avons en revanche pu déceler quelques tendances intéressantes allant dans notre sens (au niveau de la concession par exemple) mais elles seront à vérifier sur un corpus plus large et 
plus varié sur le plan des disciplines. Même les marqueurs intersubjectifs semblent plus sensibles à la différence entre disciplines qu'à la différence entre degrés de spécialisation.

Comment rendre compte de ce bilan en demi-teinte? Plusieurs explications sont envisageables:

(i) L'hypothèse de langues transversales fondées sur la notion de degré de spécialisation est fausse.

(ii) L'hypothèse est vraie mais les langues transversales ne se distinguent pas sur le plan grammatical.

(iii) L'analyse grammaticale doit être repensée.

La proposition (i) est contre-intuitive. On conçoit mal que l'ambition affichée de revues telles que History Today ou Think de s'adresser à un public non spécialisé puisse ne pas se manifester dans la réalité linguistique, d'autant qu'elle se traduit bien sur le plan lexical. Une recherche rapide sur le suffixe <-ism> , qui est le signe d'un certain niveau d'abstraction et de technicité ${ }^{6}$, révèle en effet une différence significative entre les corpus spécialisés et les textes vulgarisés et ce, dans les deux disciplines (cf. tableau 4).

Tableau 4. Fréquence du suffixe $<-$ ism > selon la discipline et son degré de spécialisation

\begin{tabular}{|l|l|l|l|l|}
\hline & \multicolumn{2}{|l|}{ PHILOSOPHIE } & \multicolumn{2}{l|}{ HISTOIRE } \\
\hline & spécialisé & non spécialisé & spécialisé & non spécialisé \\
\hline Occurrences de <-ism> & 66 & 44 & 196 & 34 \\
\hline
\end{tabular}

61 Si l'on considère un autre suffixe, <-ical>, susceptible de fournir des adjectifs dénotant des caractéristiques abstraites ou techniques (les adjectifs en <-ical> plus fréquents dans les deux sous-corpus spécialisés sont en effet philosophical, epistemological, rhetorical, metaphilosophical et metaphorical en philosophie, et political, ecclesiastical, biblical, radical et critical en histoire ${ }^{7}$ ), on retrouve exactement les mêmes tendances (cf. tableau 5).

Tableau 5. Fréquence du suffixe <-ical> selon la discipline et son degré de spécialisation

\begin{tabular}{|l|l|l|l|l|}
\hline & \multicolumn{2}{|l|}{ PHILOSOPHIE } & \multicolumn{2}{l|}{ HISTOIRE } \\
\hline & spécialisé & non spécialisé & spécialisé & non spécialisé \\
\hline Occurrences de <-ical> & 199 & 107 & 112 & 68 \\
\hline
\end{tabular}

62 On pourrait alors objecter, et c'est l'option (ii), que le degré de spécialisation n'affecte que le lexique et pas la grammaire. Outre le fait que cette position n'est pas corroborée par certains de nos résultats - fussent-ils modestes - elle est difficilement tenable sur les plans théorique et méthodologique. Comme l'a montré Langacker (1987), et comme cela a été confirmé par deux décennies de recherches en grammaticalisation (cf. Traugott \& Hopper 1993, par exemple) et dans le cadre des grammaires des constructions (cf. Goldberg 1995 ou Croft 2001), le lexical et le grammatical ne peuvent 
s'envisager que comme les pôles extrêmes d'un continuum. Prétendre que le degré de spécialisation n'affecte que le lexique conduirait ainsi à une aporie puisqu'on serait amené à postuler une frontière nette entre grammaire et lexique qui n'est pas fondée empiriquement. Une version atténuée de (ii) consisterait à dire que le degré de spécialisation n'affecte pas toute la grammaire - ce qui, d'une certaine façon, nous amène à la position (iii).

En effet, tout porte à croire que c'est en partie l'analyse grammaticale telle qu'elle a été conduite qui occulte les propriétés linguistiques des discours transversaux.

En premier lieu, le nombre nécessairement limité de marqueurs retenus dans le cadre de cette étude ne saurait suffire à dresser un tableau complet des spécificités grammaticales inhérentes aux degrés de spécialisation.

En outre, le postulat de départ est à réévaluer: évidemment il n'existe pas de marqueurs strictement référentiels ou strictement intersubjectifs. Cela n'a jamais été notre position. Mais peut-être est-il déjà excessif de parler de « dominante référentielle ou intersubjective». La distinction entre marqueurs référentiels et intersubjectifs semble difficilement tenable, à cause des chevauchements incessants entre ces métafonctions. Il apparaît aussi que certains emplois typiquement intersubjectifs sont liés à la discipline et non au degré de spécialisation (le cas des modaux est très clair : il est lié à la pratique même de la philosophie). Nous avons cherché à évaluer si les modaux étaient à cet égard trop particuliers et nous avons effectué une rapide recherche sur deux connecteurs, because et but. La figure 1 montre que ceux-ci sont effectivement sensibles au degré de spécialité, puisqu'ils prédominent en discours vulgarisé (en noir sur le schéma). Mais on observe aussi qu'ils restent sensibles à la différence entre disciplines, puisqu'ils sont de toute façon plus nombreux en philosophie qu'en histoire.

Figure 1. Répartition de because et de but dans le corpus

spécialisé

vulgarisé

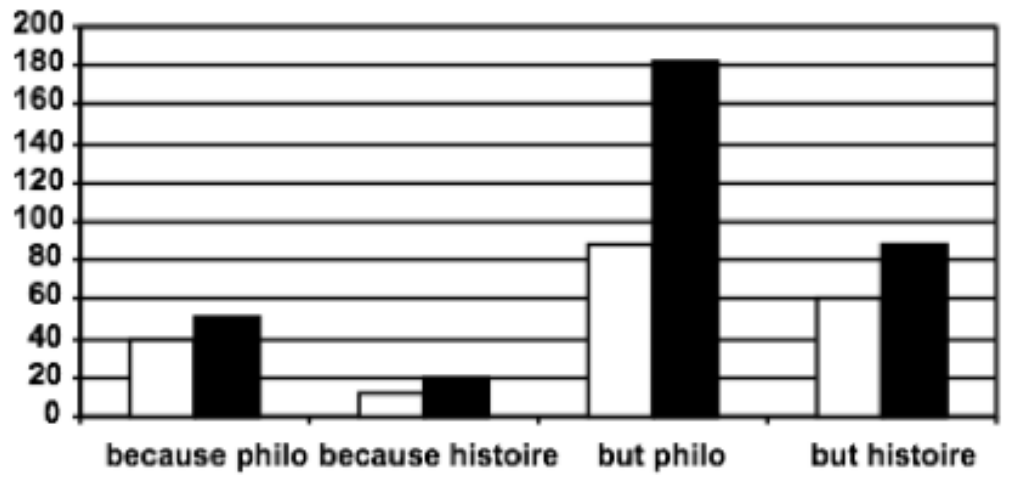

\section{Conclusion}

67 Les recherches doivent encore se poursuivre pour déterminer ou infirmer définitivement la réalité linguistique d'une grammaire liée au degré de spécialisation. 
Beaucoup reste à faire. Plus de marqueurs devront être étudiés dans le détail : pronoms personnels, tournures syntaxiques liées à la gestion de l'information dans la phrase, détermination... Un corpus plus étendu, couvrant davantage de disciplines peut-être moins «proches " que l'histoire et la philosophie, sera assemblé. Outre la taille du corpus, il faudra également être attentif aux spécificités génériques des textes. Il apparaît en effet que les sous-corpus vulgarisés, bien que constitués d'articles, présentent des variations significatives en termes de genres. On trouve, par exemple, dans les textes historiques non spécialisés, des comptes rendus d'expositions ou une balade historique dans les rues de Tallin, tandis que le sous-corpus de philosophie vulgarisée nous offre un article sous forme de narration et même un dialogue futuriste entre un robot et son créateur. Se posera alors un problème théorique complexe: faudra-t-il, pour éliminer les interférences dues à ce type d'emprunts, sélectionner uniquement des textes « purs » en termes de genre (à supposer que cela soit possible), ou au contraire, préserver cette diversité générique qui peut apparaitre comme une propriété constitutive des textes vulgarisés?

\section{BIBLIOGRAPHIE}

Adamczewski, Henri. 1982. Grammaire linguistique de l'anglais. Paris : Armand Colin.

Adams Smith, Diana. 1984 "Medical discourse: aspects of author's comment". The ESP Journal 1, $25-36$.

Banks, David. 1993. Writ in Water: Aspects of the Scientific Journal Article. Brest: ERLA, Universite de Bretagne occidentale.

Bhatia, Vijay K. 1993. Analysing Genre: Language Use in Professional Settings. Londres : Longman.

Croft, William. 2001. Radical Construction Grammar: Syntactic Theory in Typological Perspective.

Oxford : Oxford University Press.

Delmas, Claude. 1993. « De l'extralinguistique au métalinguistique ». in Lapaire J.-R. \& W. Rotgé (dir.), Séminaire pratique de linguistique anglaise. Toulouse : Presses Universitaires du Mirail, 195-212

Ghadessy, Mohsen, Alex Henry \& Robert L. Roseberry (dir.). 2001. Small Corpus Studies and ELT: Theory and Practice. Amsterdam : John Benjamins.

Goldberg, Adele. 1995. Constructions: A Construction Grammar Approach to Argument Structure. Chicago : University of Chicago Press.

Hagège, Claude. 1990. La structure des langues. Paris : Presses Universitaires de France.

Halliday, Michael A. K. 1994. An Introduction to Functional Grammar. Londres : Edward Arnold.

Halliday, Michael A. K., Angus McIntosh \& Peter Strevens. 1964. The Language Sciences and Language Teaching. Londres : Longman

Hopper, Paul \& Elizabeth Traugott. 1993. Grammaticalization. Cambridge : Cambridge University Press. 
Huddleston, Rodney. 1971. The Sentence in Written English. Cambridge : Cambridge University Press.

Jakobson, Roman. 1963. Essais de linguistique générale. Paris : Les Éditions de Minuit.

Jespersen, Otto. 1942. A Modern English Grammar on Historical Principles. Part vi. Morphology. Copenhague : Ejnar Munksgaard.

Langacker, Ronald. 1987. Foundations of Cognitive Grammar, vol.1. Stanford : Stanford University Press.

Larreya, Paul \& Claude Rivière. 2005. Grammaire explicative de l'anglais. Paris : Longman.

Lerat, Pierre. 1995. Les langues spécialisées. Paris : Presses Universitaires de France.

Magnet, Anne. 2001. «Étude de l'article scientifique de recherche en anglais : discours sur la méthode ». In Mémet M. \& M. Petit (dir.), L'anglais de spécialité en France, Mélanges en l'honneur de Michel Perrin. Bordeaux : GERAS Éditeur, 54-71.

Mémet, Monique. 2005. « Développement de l'enseignement et de la recherche en anglais de spécialité : avancées théoriques et applications pédagogiques ». Langues modernes 1, 31-38.

Mémet, Monique \& Michel Petit (dir.). 2001. L'anglais de spécialité en France. Bordeaux : GERAS Éditeur.

Resche, Catherine. 2001. "Réflexions sur la frontière entre langue générale et langue spécialisée ». In M. Mémet \& M. Petit (dir.), L'anglais de spécialité en France. Bordeaux : GERAS Éditeur, 37-46.

Mayer, Felix (dir.). 2001. Languages for Special Purposes: Perspectives for the New Millennium. Tübingen : Narr.

Richard, Isabelle. 2003. « Valeurs essentielles des modaux dans le discours juridique en anglais ». Thèse de doctorat, Université Paris 3.

Rondeau, Guy. 1983. Introduction à la terminologie. Chitoumiti, Québec : Gaetan Morin Éditions.

Sager, J.C., D. Dungworth \& P.F. Macdonald. 1980. English Special Languages. Principles and Practice in Science and Technology. Wiesbaden : Brandstetter

Sinclair, John. 2004. « Developing linguistic corpora: a guide to good practice corpus and text. Basic principles ». In M. Wynne (dir.), Developing Linguistic Corpora: a Guide to Good Practice. Oxford : AHDS, 6-23.

Swales, John. 1990. Genre Analysis. English in Academic and Research Settings. Cambridge : Cambridge University Press.

Wynne, Martin (dir.). 2004. Developing Linguistic Corpora: A Guide to Good Practice. Oxford : AHDS.

Zipf, George Kingsley. 1935. The Psychobiology of Language. New York : Houghton Mifflin.Image $200000160000005400000054315 E C E A 5$.pctImage 200000160000005400000054C5BBFEDA.pct

\section{NOTES}

1. Elle suppose toutefois une organisation radiale au sein de laquelle les genres peuvent trouver leur place (même si les genres ne sauraient se ramener au seul degré de spécialisation).

2. Nous avons bien sûr conscience que la « langue générale » est un construit théorique dont la définition est tout aussi, sinon plus, problématique que celle des langues spécialisées. 
3. Le terme est de Halliday (1994).

4. L'étiquetage a été fait par CLAWS au format C5. Ce logiciel est accessible à l'adresse <http:// ucrel.lancs.ac.uk/claws/>.

5. Le concordancier Antconc (version 2.3.1) permet de faire des recherches par mots clés et par

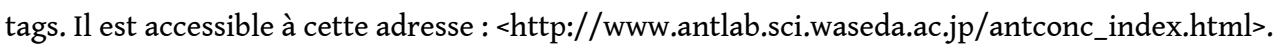
6. Jespersen (1942 : 336) qualifie en effet les noms en <-ism> d' « abstract nouns » et relève, comme emploi principal, le renvoi à «a system or doctrine or movement» (par exemple : catholicism, cisalpinism, zeolotism, dans les textes spécialisés), ce qui inclut le cas particulier d'une doctrine associée à un individu lorsque le suffixe dérivationnel est affixé à un nom propre (cf. Richerism, Febronianism ou Josephism dans le corpus spécialisé d'histoire).

7. En plus d'être moins nombreux, les adjectifs en <-ical> utilisés dans les textes vulgarisés sont moins ésotériques: pas d'axiological ou de diacritical mais plutôt des adjectifs courants comme physical (le plus fréquent en philosophie vulgarisée) ou biological.

\section{RÉSUMÉS}

Cette étude s'interroge sur la réalité de langues transversales transcendant les langues de spécialité et fondées sur la notion de degré de spécialisation. Existe-t-il une grammaire propre aux textes de vulgarisation et une grammaire propre aux textes spécialisés, quelle que soit la discipline envisagée? Quels marqueurs grammaticaux sont susceptibles de coder les différences linguistiques entre les divers degrés de spécialisation? L'hypothèse examinée est que les marqueurs référentiels (comme les temps) sont plutôt révélateurs des différences entre les disciplines, alors que les marqueurs intersubjectifs (comme les modaux) sont plutôt sensibles au degré de spécialisation d'un discours donné. L'analyse d'un corpus composé de textes spécialisés et de textes de vulgarisation en histoire et en philosophie ne confirme que partiellement cette hypothèse de départ : les marqueurs, qu'ils soient référentiels ou intersubjectifs, semblent dans tous les cas plus liés à la discipline qu'au degré de spécialisation. De nouvelles pistes sont alors proposées pour parvenir à cerner les traces linguistiques des divers degrés de spécialisation.

The present study purports to investigate the reality of cross-register discourses, i.e., discourses transcending LSPs and based on the notion of degree of specialization. Is there such a thing as a 'grammar of popularization' as opposed to a 'grammar of specialization' regardless of the subject field? Which grammatical markers are likely to encode linguistic differences between various degrees of specialization? We posit that referential markers (such as tenses) indicate differences between disciplines whereas intersubjective markers (such as modal auxiliaries) reveal differences in degrees of specialization. The analysis of a corpus made up of popularized texts and specialized texts in the fields of philosophy and history only partially confirms the hypothesis: all markers, whether referential or intersubjective, seem to depend more on the field than on the degree of specialization. Follow-up studies are suggested to eventually identify the linguistic phenomena which mark the degrees of specialization. 
INDEX

Mots-clés : degré de spécialisation, grammaire, sciences humaines, vulgarisation

Keywords : degree of specialization, grammar, humanities, popularization

\section{AUTEURS}

\section{ELSA PIC}

Agrégée d'anglais, Elsa Pic est maître de conférences en linguistique anglaise à l'Université Sorbonne Nouvelle - Paris 3. Ses recherches portent sur la terminologie, l'anglais juridique et les spécificités linguistiques des langues de spécialité. elsa.pic@univ-paris3.fr

\section{GRÉGORY FURMANIAK}

Agrégé d'anglais, Grégory Furmaniak est maître de conférences en linguistique anglaise à l'Université Sorbonne Nouvelle - Paris 3. Il a une longue expérience de l'enseignement en secteur LANSAD. Ses recherches portent sur l'aspect et la modalité en synchronie et en diachronie, ainsi que sur l'anglais de spécialité. gregory.furmaniak@univ-paris3.fr 